\title{
Theory of constructions and set in problem solving
}

\author{
JAMES G. GREENO and MARIA E. MAGONE \\ University of Pittsburgh, Pittsburgh, Pennsylvania 15260 \\ and \\ SETH CHAIKLIN \\ University of Washington, Seattle, Washington 98195
}

\begin{abstract}
Hierarchically organized knowledge about actions has been postulated to explain planning in problem solving. Perdix, a simulation of problem solving in geometry with schematic planning knowledge, is described. Perdix's planning knowledge enables it to augment the problem space it is given by constructing auxiliary lines. The planning system also provides a mechanism that can result in problem solving set. Results of three experiments involving set and constructions seem consistent with the kinds of knowledge structures hypothesized in the model. Protocols given during solution of 11 geometry problems showed general agreement with the explanation of constructions and set based on planning knowledge, but they also indicated processes of human problem solving not represented in the model. Finally, the explanation of constructions is discussed in relation to the general question of ill-structured problems and creativity, and the explanation of set is discussed in relation to other phenomena in the problem solving literature, including functional fixedness.
\end{abstract}

Several recent analyses of knowledge used in solving problems have hypothesized a structure in which knowledge about actions is hierarchical. Knowledge about each action includes knowledge about preconditions that are required for the action to be performed, knowledge about consequences of the action, and knowledge of the component subactions that are performed in the process of performing the more global action. A system based on this principle was developed by Sacerdoti (1977) in the domain of robotics, and systems designed along similar lines have been proposed for a number of other problem domains. These include an analysis of geometry theorem proving by Goldstein (Note 1), analyses of solving textbook problems in physics by Bhaskar and Simon (1977) and by Larkin (Note 2), an analysis of designing electrical circuits by Sussman (1977), an analysis of writing prose by Flower and Hayes (in press), analyses of designing computer programs by Atwood, Polson, Jeffries, and Ramsey (Note 3), Goldstein and Miller (Note 4), and Long (Note 5), and a general discussion by McDermott (1978). These theories are all consistent in a general way with earlier suggestions by Berlyne (1965), Duncker (1945), Hull (1930), Miller, Galanter, and Pribram (1960), and others regarding hierarchical structure of thinking and behavior. The more recent work has included hypotheses about the specific knowledge structures involved in performing the various kinds of tasks that have been studied.

This research was sponsored by the Personnel and Training Research Programs, Psychological Sciences Division, Office of Naval Research, under Contract N00014-78-C-0022, Contract Authority Identification Number NR 157-408.
This paper presents an analysis of hierarchical knowledge for planning in solving textbook problems in high school geometry. A simulation model of problem solving called Perdix (Greeno, 1978) has been extended in a way that involves hierarchical planning knowledge. This development has provided two extensions of previous theories. First, an explanation is provided for the occurrence of constructions in problem solving. Second, the knowledge of global actions used in planning provides an explanation of problem solving set.

The developments presented here permit a conceptual integration of several general issues in the theory of problem solving. The process developed for constructions is relevant to issues in the theory of ill-structured problem solving and the theory of creativity. There is a strong connection between constructions and set, because the main cognitive structure that causes set is also the structure that permits constructions to occur.

One feature of ill-structured problems is that important materials needed for solution are not provided in the initial problem space. Auxiliary lines needed for solving some geometry problems are materials that must be added to the problem space by the problem solver. Thus, Perdix's ability to add constructions provides some new understanding of an aspect of illstructured problem solving. In the domain of geometry, the problem solving demands are relatively modest in comparison, for example, with musical composition (Reitman, 1965). Nonetheless, the kind of process that enables Perdix to construct auxiliary lines seems applicable in other domains as well.

The process represented in Perdix for augmenting a problem space involves a form of problem finding. 
Problem finding has been identified as a major factor in creative work on ill-structured problems such as painting (Getzels \& Csikszentmihalyi, 1976). Thus, the theoretical development to be presented may provide a step toward a more rigorous analysis of some aspects of creative processes in problem solving.

The explanation of set that is provided by hierarchical knowledge of actions is entirely consistent with conventional explanations of set in experimental psychology (e.g., Bourne, Ekstrand, \& Dominowski, 1971). The representation in Perdix specifies knowledge structures for solving a class of problems in a way that shows how set can occur. The point of greatest interest is the close relationship between the cognitive structures involved in set and constructions. The analysis also suggests some ways to begin to interpret the considerable experimental literature on problem solving set in relation to recently developed theoretical concepts of information processing and representation of knowledge.

We will begin by presenting illustrations of the phenomena of constructions and set in geometry problem solving, in order to provide a concrete context for the theoretical concepts that we will develop. Next, we will describe the knowledge used by Perdix for planning and the way in which Perdix simulates phenomena of constructions and set. Then, we will describe the results of three experiments and a set of thinking-aloud protocols that provide further illustrations of the principles that are incorporated in the model, and we will also indicate some important aspects of human processing involving constructions that are beyond the capabilities programmed in Perdix. Finally, we will discuss general implications of the analysis, concerning the relation of constructions to the issues of ill-structured problems and creativity and the general question of set in problem solving.

\section{Example of Construction}

An example of a problem that is solved with a simple construction is the following: Prove that if two sides of a triangle are congruent, then the angles opposite those sides are congruent. (An alternative statement is: Prove that the base angles of an isosceles triangle are congruent.)

Table 1 shows a protocol of a solution of this problem. The student began by drawing a triangle, labeling its vertices $A, B$, and $C$, marking the congruent sides $\overline{\mathrm{AC}}$ and $\overline{\mathrm{BC}}$, and marking the angles at $\mathrm{A}$ and $\mathrm{B}$, which were to be proved congruent, as shown in Figure 1. At ${ }^{*} 1$ in the protocol, the student drew a line from $\mathrm{C}$ to the base of the triangle, labeling the point of intersection $\mathrm{D}$. At ${ }^{*} 2$ the student tentatively specified that $\overline{C D}$ would be the bisector of $\angle A C B$ and confirmed this at $* 3$, when the student realized that, since $\overline{C D}$ is in both triangles, it provides the needed second pair of congruent sides for the side-angle-side pattern of congruence of triangles.
Table 1

Protocol for the Base Angles Theorem

S: Okay, so, I want to prove that angle $A$ is congruent to angle $B$. Now, let's see. Do you want ... ?

*1 E: Yeah ... why are you drawing that line?

S: I don't know yet. Okay, uhm ... okay, then I could ... if I drew a line ...

E: $M m-h m m$

*2 S: That would be the bisector of angle ACB, and that would give me ... those congruent angles ... no. (Pause)

*3 Yeah, well that would give me those congruent angles, but I could have the reflexive property, so this would be equal to that. Okay, I got it.

E: Now, before you go ahead and write it all down, when you said you were going to draw the line...

S: Yeah ...

E: And I said, why are you doing that, and you said you didn't know yet, what do you think happened to give you the idea of making it the bisector?

*4 S: Okay. Well, I have to try to get this ... I have to try to get triangle $A C D$ congruent to $B C D$, because if $I$ do that, then angle $A$ is congruent to angle $B$ because corresponding parts of congruent triangles are congruent.

E: So you were drawing the line to give yourself triangles, is that the idea?

*5 S: No, to ... to get a side that was in both triangles.

E: Okay.

*6 S: And to get congruent angles.

E: So that's why you drew it as the bisector.

S: Yeah.

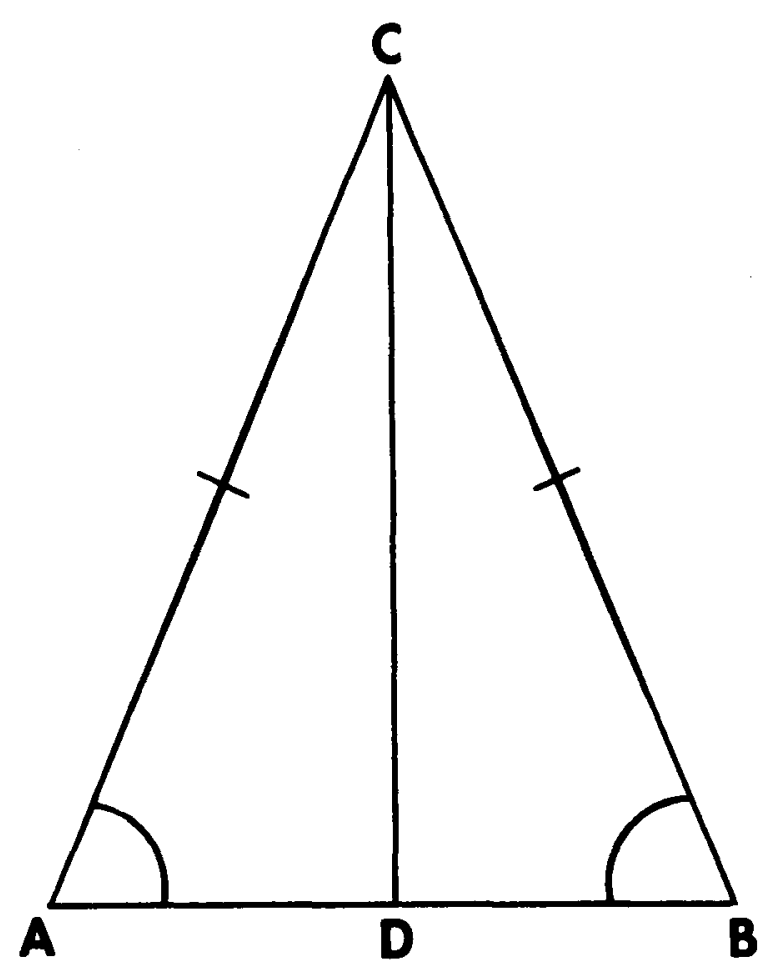

Figure 1. Diagram drawn by student during solution given in Table 1. 


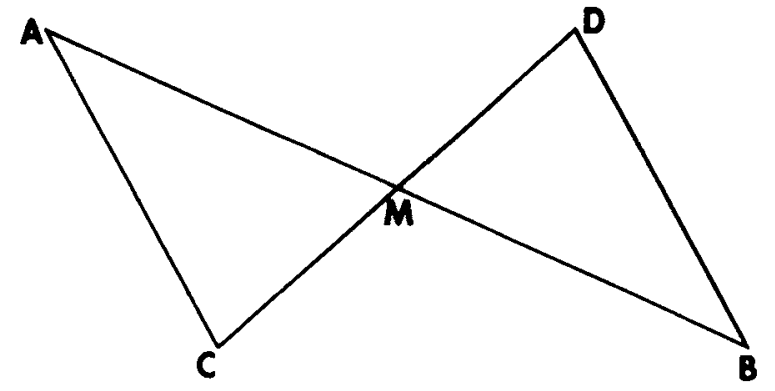

Given: $M$ is the midpoint of $\overline{A B}$ and $\overline{C D}$; $\overline{\mathrm{AC}} \cong \overline{\mathrm{B}} \overline{\mathrm{D}}$

Prove: $\angle A M C \cong 1 . B M D$

Figure 2. Problem of the kind studied by Luchins (1942).

\section{Example of Set}

Consider the problem shown in Figure 2. The problem can be solved by proving that triangles are congruent: $\triangle \mathrm{ACM} \cong \triangle \mathrm{BDM}$ (side-side-side); then $\angle A M C \cong \angle B M D$, since they are corresponding parts of congruent triangles. However, an easier solution is possible. $\angle A M C$ and $\angle B M D$ are vertical angles, so their congruence can be inferred directly.

Luchins (1942) gave a problem similar to the one in Figure 2 to 10 student subjects who had just solved a series of four problems in which congruence of angles was shown by proving that triangles were congruent. Eight of the 10 students solved the problem by proving that triangles were congruent, rather than by the simpler solution involving vertical angles. Another set of 10 students solved four problems involving congruence of triangles, followed by a problem similar to Figure 1, in which the given information was insufficient to prove the triangles congruent. Six of these 10 students failed to find a solution to the problem in the $2.5-\mathrm{min}$ period that was allowed. In contrast, the problem was solved easily by all 12 students in a group that was given the problem without preceding problems to induce the attempt to prove triangles congruent.

\section{GENERAL HYPOTHESES}

The gist of the explanation of set and constructions to be proposed here is that both are consequences of the way in which planning occurs. The hypothesis to be proposed about planning is a hierarchical system that includes a set of plan-schemata that are associated with subgoals. Each plan-schema includes information about global features of the situation that are required for the plan to be feasible; for example, the plan of proving triangles congruent requires that two triangles be present in the diagram. When a plan has been adopted, work proceeds in relation to the specific subgoals that are required for that plan to succeed.

This planning system produces constructions by a process of partial pattern matching and pattern comple- tion. Perdix recognizes that a situation partially matches a pattern that satisfies the prerequisites for a planschema and then activates productions that complete the required pattern. For example, a pattern that frequently enables the plan of proving congruence of triangles is a pair of triangles with a shared side. Before line $\overline{C D}$ is drawn in Figure 1, the diagram contains some, but not all, of the lines, angles, and points that constitute that pattern. A system that can detect that partial match and identify the discrepancy between the situation and the pattern it has in memory has the knowledge it needs to choose an appropriate construction for this problem.

This idea also provides an explanation of problem solving set. A plan may be adopted on the basis of global features, and because of the top-down nature of the system, the plan will become the basis of work on the problem until either the plan succeeds or it is determined that the plan is impossible. For example, in Figure 2 the prerequisites are present for the plan of proving that angles are congruent by showing that they are corresponding parts of congruent triangles. If this plan is adopted, the system focuses on the subgoal of proving congruence of the triangles and can easily fail to notice that the angles are vertical angles.

\section{PLANNING KNOWLEDGE IN PERDIX}

We will give a brief survey of the characteristics of Perdix as a problem solving system. More detailed information about Perdix's geometry knowledge has been given in other articles (Greeno, 1976, 1977, 1978).

Perdix is a system that solves problems by matching and completing patterns. It is a production system with a general control structure adapted from Anderson's (1976) ACT system. The problem situation is represented as a semantic network. Points, lines, angles, and closed figures are represented by nodes. Connections between nodes include part-whole relations (e.g., a point that is part of a line segment or a segment that is part of a triangle) and propositional structures that correspond to relations of congruence and other geometric relations.

The productions that constitute Perdix's problem solving knowledge are in three categories: inferential principles, feature tests for pattern recognition, and strategic knowledge. The main problem solving steps are accomplished by inferential productions. These represent knowledge of propositions such as "Vertical angles are congruent," or "Angles formed by bisection of an angle are congruent." The actions of these productions add relations to the semantic network that represents the situation. For example, if two angles have previously been identified as vertical angles, the relation of congruence can be added between them.

Simple pattern recognition is involved in the conditions that are tested in each production. More complex patterns are identified by sets of productions organized in the form of decision networks with terminal nodes corresponding to patterns and relations that can be identified. Patterns and relations that are identified 
during problem solving are added to the representation of the problem situation.

Strategic knowledge consists of productions for setting goals and adopting plans. Simple goal setting involves associations that select productions that are relevant to the current problem solving goal. More complex goals are set in the actions of productions, and a push-down store memory is used to retain goals that must be recalled when their subgoals have been achieved.

The theoretical development to be reported in this paper is a set of productions that perform planning. Perdix's knowledge for planning consists of some global actions, or plan-schemata. The characteristics of planschemata are like those used by Sacerdoti (1977) in his theory of planning based on a procedural network. Goldstein (Note 1) used a similar organization of knowledge for geometry problems; his system includes experts that apply knowledge in specific topic areas, such as triangles or parallel lines. Each plan-schema in Perdix is characterized by three components of informa. tion: (1) a set of prerequisite features that make the plan promising, (2) pointers to pattern recognition and inferential productions that are used in executing the plan, and (3) tests to determine whether execution of the plan has succeeded. The procedures for planning also include checks for a few salient features that would allow the goal to be achieved directly without going through the usual process of planning and executing a series of steps. Technically, these shortcuts could be classified as additional plans.

In Perdix's knowledge structure, plans are associated with general goals. Some goals are attempted directly through productions for pattern recognition. Other goals require propositional inferences. The latter goals activate planning productions. When one of these goals is generated during problem solving, a series of tests is performed. Each test is an attempt to find the general features that make one of the plans for the goal promising. Each planning system is thus a pattern recognizer in which each identifiable pattern corresponds to a set of features needed to make one of the plans promising.

Two planning systems have been implemented in Perdix. One is associated with the goal of finding the measure of an angle, and the other is associated with a category of goals of finding or proving a quantitative relationship such as congruence between two angles or two segments. One plan for finding the measure of an angle uses the sum of angles in a triangle. The other uses a quantitative relationship with another angle. The planning system is essentially a decision tree that chooses one of these plans, based on whether the unknown angle is part of a triangle and on the nature of available information about the measures of other angles in the situation.

We will describe the planning system for inferring quantitative relations in some detail. Perdix has three plans for inferring quantitative relations between angles or segments. It can infer congruence between angles or segments that are corresponding parts of congruent triangles. This plan is abbreviated TRICONGQREL: TRlangle CONGruence is used to infer a Quantitative RELation. A second plan uses relations between angles with a shared vertex: RTNRELQREL means that a relation based on rotation, RTNREL, is used to infer a Quantitative RELation. The third plan uses relations between angles with parallel sides: PRLRELQREL.

The procedure that has been implemented for constructing auxiliary lines is part of the planning system for quantitative relations. If none of the preconditions for Perdix's three main plans is found, Perdix can identify a partial match to a pattern that provides the prerequisites for one of its plans, the congruence of triangles. If a sufficient subset of the features of that pattern is present, Perdix completes the pattern and will then choose the plan that its own construction has enabled.

Figure 3 shows the components of the planning procedure for finding a quantitative relation between two angles or segments. V1 and V11 denote the angles or segments in that goal. There are tests at Steps 1 , 2 , and 3 for a few features that can provide quick solutions. Next, at Step 4, Perdix tests whether the target elements are parts of triangles. If they are, triangles are tested at Steps 5,6, and 7 to see whether there are features that would make it feasible to try to prove that they are congruent. These tests have two functions. First, if the triangles are not promising, the system can look for other plans that might be easier. Second, since many diagrams include more than one pair of triangles, the tests at Steps 5,6 , and 7 serve to select triangles that contain congruent components. If one of these tests is successful, then, at Step 8, the plan is adopted to find a quantitative relation based on congruent triangles, and at Step 9, the goal is set to show that the selected triangles are congruent.

If promising triangles are not found, Perdix tests at Steps 10 and 13 for features of its other general plans for finding quantitative relations. If the targets are angles with a single vertex, then at Step 11, the plan RTNRELQREL is adopted to infer a quantitative relation from a relation based on rotation, and at Step 12, the goal is set to find a relation of rotation between the angles. The system will then engage in pattern recognition that can identify vertical angles, linear pairs, or angles formed by perpendicular lines. If the test at Step 13 shows that the angles are formed by parallel lines intersected by a transversal, then at Step 14, the plan PRLRELQREL is adopted to infer a quantitative relation from a relation involving parallel sides. Under the goal PRLREL, set at Step 15, Perdix will perform pattern recognition that can identify corresponding angles, alternate interior angles, or interior angles on the same side of the transversal. If these plans are not feasible, Perdix will try to use 


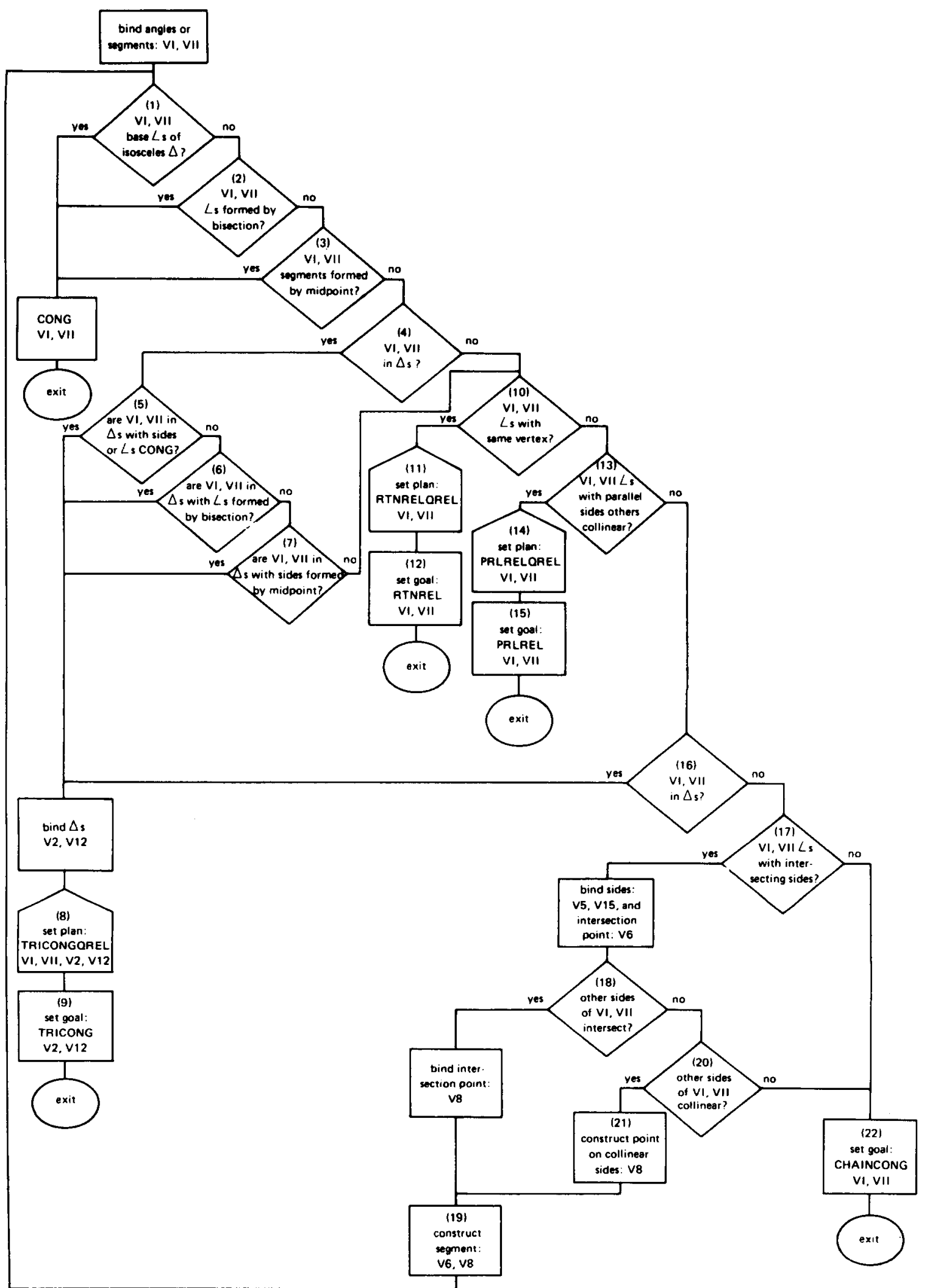

Figure 3. Procedure for planning, including construction of new components, when goal is to find a quantitative relation between angles or segments. 
triangles that seemed unpromising earlier, if they are found in the test at Step 16.

The process for constructing auxiliary lines is used if the test at Step 16 is negative. If the prerequisite features of none of the available plans are present, Perdix checks whether the problem situation has features that provide a partial match for a schema that includes the prerequisites for one of the plans. The schema consists of a pair of triangles with a shared side. It contains 11 components: two triangles, five segments, and four points. The partial match can consist of two segments that intersect, found at Step 17, and either of two further conditions: two additional intersecting segments at Step 18 or a single segment found at Step 20 that can be divided to form two needed segments. If the appropriate components are found, Perdix constructs a segment that completes the pattern at Step 19 and returns to the top of the planning process.'

If the features needed to perform this construction are not present, then the system adopts the goal of finding a chain of relations between angles at Step 22 . This is a kind of default plan, involving an attempt to find some third angle that is congruent to one of the angles that can then be related to the other target, perhaps through mediation of further linking angles.

When the planning process is finished, one of three things will have happened. First, the goal may have been achieved on the basis of a feature permitting a quick solution. Second, a plan may have been chosen; this generally involves setting a subgoal that will permit the plan to work. Adoption of a plan may have been preceded by a construction to make the plan feasible. Finally, the system's planning knowledge may be insufficient for the problem at hand, and there may have been no successful outcome for planning. (In the system shown in Figure 3, this leads to setting the goal of forming a chain of congruent components.) In the situation in which a plan is chosen, the system then executes procedures that are associated with the plan, including pattern recognition and propositional inferences.

\section{EXPLANATION OF CONSTRUCTIONS AND SET}

The planning process in Perdix is an example of one kind of knowledge structure that can produce constructions and set. The relevant general feature is the knowledge of global actions and planning systems that choose a general approach to the problem from among the available global actions. Figure 3 is an example: The alternative global actions are called TRICONGQREL, RTNRELQREL, and PRLRELQREL. The planning system uses general features of the problem situation, such as the presence of triangles with known relations between their components, to select one of the global actions as a plan.

The general idea about constructions illustrated in Perdix involves knowledge of prerequisite features for plans and the ability to recognize partial satisfaction of a set of prerequisites. When Perdix finds that the prerequisites for none of its plans are satisfied, it can recognize that the situation has some of the components of a pattern that permits TRICONGQREL to be used. Perdix has problem solving procedures that permit it to complete this pattem and thus to produce the prerequisites that are required for its planning system to choose a global action. This illustrates a general kind of process in which knowledge of needed prerequisites permits a system to identify an auxiliary problem. The new problem arises because there is a discrepancy between this situation and conditions known to be prerequisites for making progress on the main problem. In a hierarchical system, a deficit that arises at the level of global actions can generate an auxiliary problem that is solved in a problem space different from the space of the main problem. In the geometry example, the operators for the main problem are inferential propositions, such as "Vertical angles are congruent." The operators in the auxiliary problem space are a set of rules for adding lines to a diagram, such as "Two points may be connected by a line segment," or "The bisector of an angle can be constructed." The process of identifying a deficit in the main problem space constitutes a form of problem finding and thus provides some insight into creative problem solving (Getzels \& Csikszentmihalyi, 1976) and solution of ill-structured problems (Simon, 1973).

The occurrence of set is explained in a straightforward way by the top-down nature of the planning system. Consider the problem shown in Figure 2. The two alternative solutions correspond to different plans, one involving congruent triangles and the other involving a relation between angles having the same vertex. Whether Perdix finds the simple solution involving vertical angles or proves that triangles are congruent depends on the order in which the productions testing feasibility of these plans are included in the production list. In the configuration shown in Figure 3, the features needed for the plan of congruent triangles are tested before the feature needed for the plan of a relation involving rotation. With the productions ordered in this way, Perdix agrees with the majority of Luchins' (1942) subjects on the way to solve problems similar to Figure 2. The test "V1, V11 in triangles?" passes, as does the subsequent confirming test, "V1, V11 in triangles with sides or angles congruent?" This leads to adoption of the plan to prove the triangles congruent, and the system works on that subproblem and eventually succeeds in solving the problem by proving the triangles congruent by side-side-side.

The relative strengths of productions are represented in Perdix by the order in which their conditions are tested. For example, the test for "V1, V11 angles with shared vertex?" could be performed before "V1, V11 in triangles?" In that case, Perdix would find the simpler solution based on vertical angles. The mechanism involving ordered productions is not intended as a plausible hypothesis about the way in which stronger 
productions dominate performance; other systems such as Anderson's (1976) version of ACT or the OPS system (McDermott \& Forgy, 1977) include a variety of mechanisms that have greater psychological plausibility. It would be natural to assume that the strength of a production would be increased by the major factors that are usually assumed to influence set, such as the frequency and recency of successful use of a plan in problems similar to the present one. The present analysis does not provide any new ideas about the way in which set occurs. The contribution of this analysis is in showing that the knowledge structures responsible for set may be the same as those that are responsible for planfulness and creativity in problem solving.

\section{EXPERIMENTS ON CONSTRUCTIONS AND SET}

The model described above provides a way of interpreting the results of three experiments we have conducted. The first experiment involved a problem requiring a simple construction. The problem was presented in two different forms; in one form the construction was produced by a greater proportion of subjects than in the other. A plausible interpretation of the difference between the two forms can be given in terms of the planning model that we have presented.

The second experiment used the same problem as Experiment 1. We investigated conditions that could be expected to make it more likely that subjects would produce the construction needed for solution in the harder version of the problem. One of the conditions that we used was successful in making that version of the problem easier, and the effect is consistent with the idea of pattern-based planning that is incorporated in Perdix.

The third experiment was a traditional study of set, focused on a problem requiring a construction. We used a problem that has two solutions, one based on parallel lines and the other based on congruent triangles. We gave different groups of subjects different series of problems before presenting the ambiguous problem. The problems in one induction sequence used parallel lines, and the problems in the other induction sequence used congruent triangles. The majority of the solutions that subjects chose for the ambiguous problem agreed with the plan they had been using, and this result agrees with the interpretation of problem solving set that we have given based on the model of schematic planning presented above.

\section{Experiment 1}

The experiment involved two problems, presented to different groups of subjects. Both problems used the diagram shown in Figure 4. Point $\mathrm{O}$ is the center of the circle, and $\overline{\mathrm{BC}}$ is a diameter. In the problem given to one group of subjects, given information included the measure of arc $\mathrm{AC}, 80 \mathrm{deg}$, and the goal was to find the measure of the angle $\angle A B C$. In the other form, the

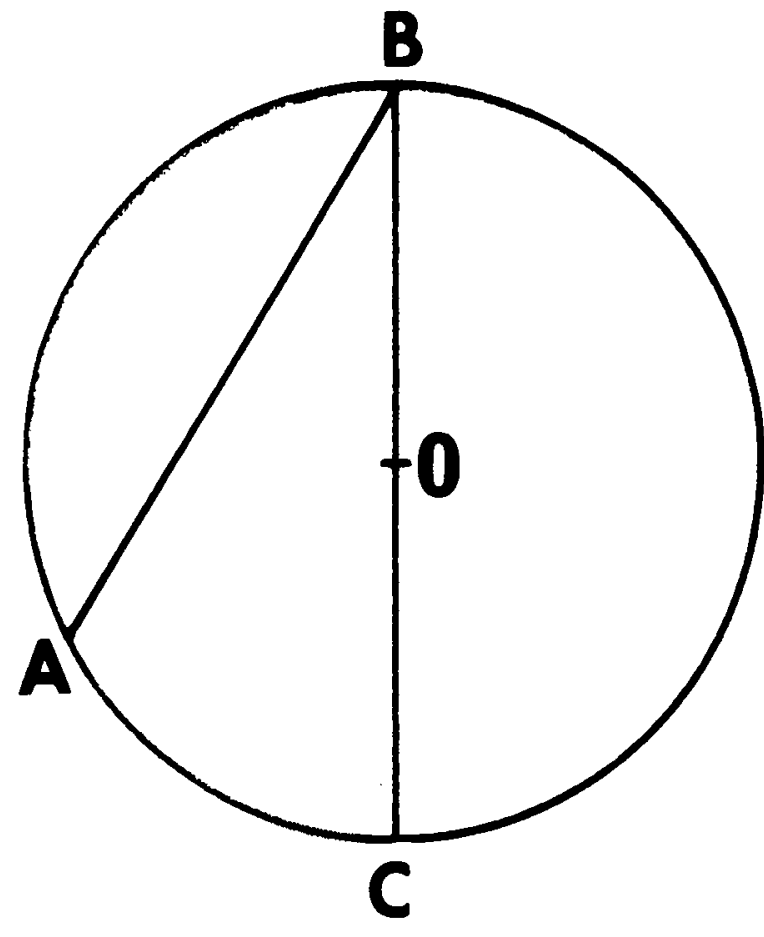

Figure 4. Diagram for problems in Experiments 1 and 2.

measure of $\angle A B C$ was given as $40 \mathrm{deg}$, and the goal was to find the measure of arc $\mathrm{AC}$. To solve either problem, a subject had to give a series of steps justifying each inference, as is customary in high school geometry. The solution of either problem depends on making the construction $\overline{\mathrm{AO}}$. This creates the central angle $\angle \mathrm{AOC}$, and, by definition, the measure of a central angle and the measure of its intercepted arc are the same. Construction of $\overline{\mathrm{AO}}$ also creates an isosceles triangle $\triangle \mathrm{OAB}$, with sides $\overline{\mathrm{OA}}$ and $\overline{\mathrm{OB}}$ that are congruent, being radii of the circle. The angles at $A$ and $B, \angle O A B$ and $\angle A B C$, are congruent, since they are the base angles of the isosceles triangle. Using these relationships, a solution for problems with the arc given is as follows: (1) Measure of arc AC is $80 \mathrm{deg}$ (given), (2) construct segment $\overline{\mathrm{AO}}$, (3) measure of $\angle A O C$ is $80 \mathrm{deg}$ (central angle), (4) measure of $\angle A O B$ is $100 \mathrm{deg}$ (linear pairs are supplementary), (5) sum of measures of $\angle O A B$ and $\angle A B C$ is $80 \mathrm{deg}$ (sum of angles in a triangle is $180 \mathrm{deg}$ ), and (6) measure of $\angle A B C$ is $40 \mathrm{deg}$ ( $\angle O A B$ and $\angle A B C$ are equal). $A$ solution for the problem with the angle given is as follows: (1) Measure of $\angle A B C$ is 40 deg (given), (2) construct segment $\overline{A O}$, (3) measure of $\angle O A B$ is $40 \mathrm{deg}$ (base angles of isosceles triangle are equal), (4) measure of $\angle A O B$ is $100 \mathrm{deg}$ (sum of angles in a triangle is $180 \mathrm{deg}$ ), (5) measure of $\angle A O C$ is $80 \mathrm{deg}$ (linear pairs are supplementary), and (6) measure of arc $A C$ is $80 \mathrm{deg}$ (arc intercepted by central angle).

\section{Method}

Subjects were students in introductory psychology at the University of Pittsburgh who participated to fulfill a course requirement. Fifty-three subjects were given the problem with the angle given, and 52 subjects were given the problem with 
the are given. Subjects participated in groups of up to six in size. Experimental sessions began with a review of geometric concepts needed to solve either problem, including the congruence of base angles of an isosceles triangle, the relation between an exterior angle and its opposite interior angles in a triangle, and the definitional equality of the measure of a central angle and the arc it intercepts on a circle. The review of concepts was given in the form of 10 problems, which were worked individually by the subjects in the group. The experimenter monitored the subjects' work and explained how to solve any problems that any subjects were unable to solve. The experimenter also called attention to features of the various training problems that were relevant to the critical problem. The 11 th problem in the series was Figure 4, with either the measure of arc $A C$ or the measure of $\angle A B C$ given and the other unknown. No assistance was given on this problem, and a period of $4 \mathrm{~min}$ was allowed for its solution. At the end of the allotted time, subjects who had not solved the problem were asked to turn to a second sheet containing the same problem, and they were given the hint that a line drawn between Points $A$ and $O$ would be helpful in solving the problem. An additional 4-min period was allowed to see whether the subjects could solve the problem with the hint given.

\section{Results}

The problem with the arc given was considerably easier than the problem with the angle given; proportions of subjects solving the problem in the initial $4 \mathrm{~min}$ were .731 and .471 , respectively. The difference is statistically reliable $\left[\chi^{2}(1)=7.34, p<.01\right]$.

An important question for our theoretical interpretation involves the degree to which the difficulty of the problem with the angle given was caused by a difficulty in seeing the correct construction. Of the 28 subjects who had the angle given and failed to solve the problem, 7 drew the correct construction during the initial 4 min of work; of the 14 subjects who had the arc given and failed to solve, 2 drew the correct construction during the initial $4 \mathrm{~min}$. If these subjects are counted along with the subjects who solved, the difference between the conditions is attenuated and becomes marginally nonsignificant $\left[.769\right.$ vs. $\left..604 ; \chi^{2}(1)=3.32, .05<p<.10\right]$. However, the status of these unsuccessful constructions is unclear. Only two subjects showed evidence in their protocols of relating the construction to appropriate relational patterns (one of the seven subjects with angle given mentioned the isosceles triangle, and one of the two subjects with arc given mentioned the central angle). An alternative interpretation is that some subjects who failed to see the correct pattern simply drew an additional line in the diagram somewhere, and the significance of the constructed line was not evident in many cases. It may be noted that irrelevant constructions were inserted by nine subjects: A radius perpendicular to the diameter was drawn by seven subjects (six with angle given, one with arc given), and Points $A$ and $C$ were connected by two subjects (one in each condition).

There was also some marginal evidence that the problem with the arc given was easier than the problem with the angle given when the construction was given as a hint. Thirteen of the 14 subjects who had the arc given and failed to solve in the first $4 \mathrm{~min}$ did solve after the hint was given; 20 of the 28 initial nonsolvers who had the angle given solved the problem after the hint was given. The difference was marginally nonsignificant $\left[.929\right.$ vs. $\left..714 ; \chi^{2}(1)=3.18, .05<\mathrm{p}<.10\right]$.

\section{Discussion}

The major finding of the experiment was that the problem with arc given was easier to solve than the problem with the angle given. The most reasonable conjecture is that the difficulty had two sources: First, subjects were apparently somewhat less likely to generate the needed construction when the angle was given; second, the sequence of inferences was apparently harder to find with the angle given, even when the segment $\overline{\mathrm{AO}}$ was present in the problem situation.

An interpretation of the result can be given using the idea of schematic planning. A relevant fact is that student subjects usually work forward in geometry problems. Subjects who were given the measure of the arc probably tried to find features of the problem related to the arc, and subjects who were given the measure of the angle probably tried to find features of the problem related to the angle. It seems reasonable to suppose that the pattern of the central angle was relatively easy to retrieve with the arc as a cue. The measure of the arc and the measure of the central angle are related definitionally, and the central angle is a rather simple pattern. On the other hand, it was probably less likely that the pattern of the isosceles triangle formed by radii would be retrieved with the inscribed angle as a cue. There is no direct definitional connection between the measure of the inscribed angle and the triangle formed by radii of the circle, and that pattern is somewhat more complex than the inscribed angle. Thus, it seems reasonable that the greater difficulty of the problem with the angle given was caused at least partly by a lower probability of retrieving the pattern from memory that was needed to provide a basis for planning the first major step in the solution of the problem.

\section{Experiment 2}

This study explored two ways of providing experience that might facilitate solution of the harder form of the problem of Figure 4. One way to make a solution more likely would be to increase the probability of retrieving the pattern involving an isosceles triangle formed by radii of the circle. A second possibility would be to induce a strategy of planning backward from the goal of the problem, this could lead subjects to consider problem components that can be related to the measure of the arc, and thus to retrieve the pattern involving the central angle.

\section{Method}

Three conditions were used. A control condition was identical to the condition used for the problem with the angle given in Experiment 1. For the other two conditions, the sequence of review problems was condensed to seven problems, and three additional problems were included. For the isosceles pattern 
condition, the three added problems involved isosceles triangles that were formed by radii of circles. For the backward planning condition, the three added problems involved solution sequences that were easier to find by working backward from the goal than by working forward from the given information. In the backward planning condition, the experimenter specifically instructed the subjects to find a sequence of steps that started with the unknown quantity and progressed toward the given information and coached them so they identified appropriate sequences for the three problems.

The procedure was the same as in Experiment 1, with the problem of Figure 5 presented as the 11 th problem in all three conditions, with the measure of $\angle A B C$ given and the measure of arc $\mathrm{AC}$ the goal. Two groups of subjects participated: 86 students from introductory psychology at the University of Pittsburgh, who participated to fulfill a course requirement, and 81 students who answered an advertisement in the campus newspaper, who were paid for their participation. The numbers

(a)

Opposite sides of a parallelogram are equal and parallel

Show that opposite angles are equal, that is, $i A=\angle D$.

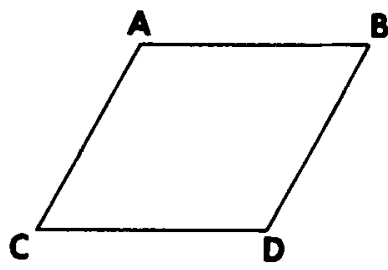

(b)

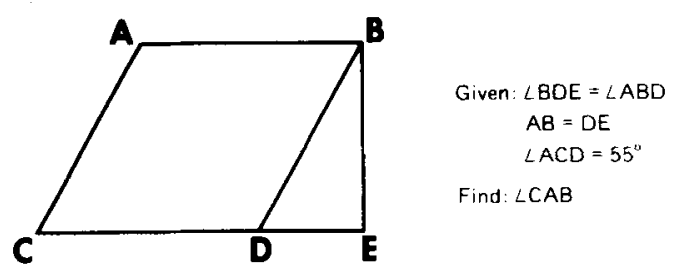

(c)

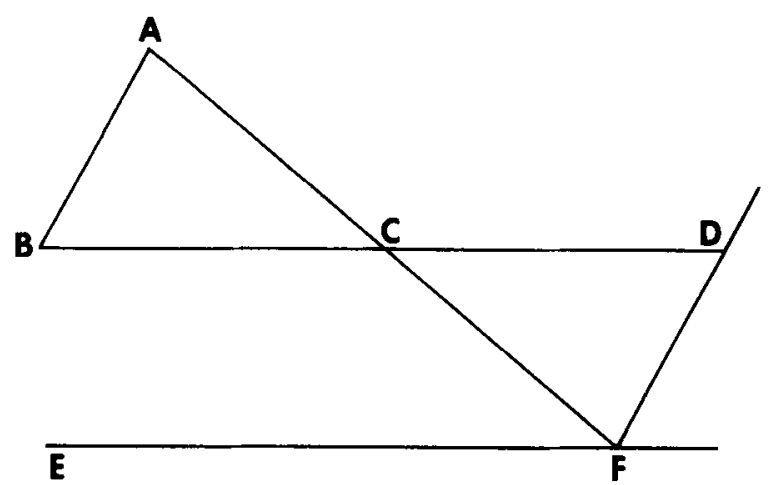

$$
\begin{aligned}
& \text { Given: } \overleftrightarrow{B D} \| \overrightarrow{E F} \\
& \overline{B D} \text { and } \overline{A F} \text { bisect each othe } \\
& \angle \mathrm{CFO}=65^{\circ} \\
& \text { i } \mathrm{ABC}=75 \\
& \text { Find: } A C B
\end{aligned}
$$
set.

Figure 5. Problems used in experiment on constructions and of subjects from these two sources in the three conditions were as follows: control, 28, 27; isosceles pattern, 29, 28; backward planning, 29, 26.

\section{Results}

The proportions of subjects who solved the problem in the three conditions were as follows: control, .436; isosceles pattern, .649; backward planning, .527. The difference was not quite large enough to give a significant test statistic $\left[\chi^{2}(2)=5.14, .05<p<.10\right]$. However, a chi-square statistic involving just the control and isosceles pattern conditions was significant $\left[\chi^{2}(1)=\right.$ $5.10, p<.025]$, and the results for the two groups of subjects were very consistent, providing some additional confidence. Proportions of solvers from introductory psychology were $.500, .724$, and .586; proportions of paid subjects who solved were $.370, .571$, and .462 . It seems reasonable to conclude that solution was made more probable in the isosceles pattern condition. A conclusion is not warranted regarding the backward planning condition; it may have provided no facilitation, or it may have facilitated problem solving as much as the isosceles pattern condition.

A substantial number of nonsolvers added the appropriate construction to the diagram, although they failed to solve the problem using it. Of the 31 nonsolvers in the control group, 15 added $\overline{\mathrm{AO}}$ to the diagram in the first 4 min of work. Of the 26 nonsolvers with backward planning induction, 9 added $\overline{\mathrm{AO}} ; 6$ of the 20 nonsolvers with the isosceles pattern added $\overline{\mathrm{AO}}$. The proportions (including solvers) who made the correct construction were clearly not significantly different: control, .709; isosceles pattern, .754; backward planning, .691. Again, the status of the correct constructions by nonsolvers is uncertain. Thirteen of the 30 correct constructions made by nonsolvers were accompanied by additional lines in the diagram that were not useful in the solution, and 21 of 47 subjects who did not make the correct construction added some line or lines in their attempts to solve the problem. It seems likely that relatively haphazard modifications of a diagram can be made by subjects, and some of the constructions in our experiments must have been of that nature. Perhaps the best interpretation of the results is that training with the isosceles pattern increased subjects' ability to identify the pattern and thus to produce a well motivated construction.

The effect of giving the hint to draw line $\overline{\mathrm{AO}}$ was inconclusive. Proportions of initial nonsolvers who solved the problem following the hint were $.613, .750$, and .654 . The result is not inconsistent with the idea that the backward planning and isosceles pattern conditions facilitated problem solving with the completed diagram as well as facilitating making the construction, but the difference was far too small to be significant $\left[\chi^{2}(2)=1.03, \mathrm{p}>.50\right]$.

\section{Discussion}

The facilitation of problem solving in the isosceles 
pattern condition is consistent with the hypothesis of a planning system that is based on knowledge of patterns that are identified as conditions for adopting plans and that are used in making constructions that take the form of pattern completion. Our interpretation is that the additional problems given with the isosceles pattern made that pattern more salient for subjects, and thus made it more likely that they would retrieve that pattern from memory in the problem situation.

\section{Experiment 3}

This experiment involved the role of problem solving set in a problem requiring a construction. Our explanation of problem solving set given above is that the pattern-based planning system becomes biased toward choice of a plan that has been successfully used in recent problems. Our explanation of constructions is that a pattern associated with a plan is partially identified, and the system completes that pattern in order to have the prerequisites for a plan. This suggests that if a problem can be solved with alternative constructions related to different plans, the construction that is chosen should be influenced by a series of problems that are solved using one or the other of the two plans.

The problem used in the experiment is shown in Figure 5a. The problem can be solved by showing that triangles are congruent. This requires constructing the diagonal line $\overrightarrow{\mathrm{BD}}$; the resulting triangles are congruent by side-side-side. The problem can also be solved using relations based on parallel lines. This solution usually uses a construction such as extending line $\overline{\mathrm{BD}}$ upward; in this case, $\angle \mathrm{A}$ is congruent to the exterior angle at $\mathrm{B}$ (alternate interior angles), and that angle is congruent to $\angle D$ (corresponding angles).

\section{Method}

The subjects were 12 University of Michigan students who were paid for participating. Thinking-aloud protocols were tape-recorded to permit later identification of subjects' solution strategies. The experimental sequence began with a series of review exercises that reminded the subject of ideas involved in proving congruence of triangles and in proving theorems involving angles formed by parallel lines and an intersecting transversal. Then there was a series of 14 induction problems. For six subjects, the induction problems all used congruent triangles; the other six subjects had 14 problems that used relations between angles based on parallel lines. Problem 15 for all subjects was the problem shown in Figure 5a. After this ambiguous problem, each subject received a problem that required use of the plan that had not been used in the induction sequence that the subject had received. These problems did not require constructions, but they gave further opportunity to observe the biasing effect of problem solving set at the level of general planning. For subjects who had been induced to use congruent triangles, Problem 16 was the one shown in Figure 5b. This is an easy problem using parallel lines: $\overline{\mathrm{AB}}$ and $\overline{\mathrm{CE}}$ are parallel ( $\angle B D E$ and $\angle A B D$ are alternate interior angles); hence, $\angle A C D$ and $\angle C A B$ are supplementary (interior angles on the same side of the transversal). Problem 16 for subjects who received induction of parallel lines is shown in Figure 5c. The triangles are congruent by side-angle-side, because of vertical angles; then, $\angle B A C=65 \mathrm{deg}$ (congruent to $\angle C F D$ ), and $\angle A C B$ can be found by subtraction from 180 deg. For the subjects with (a)

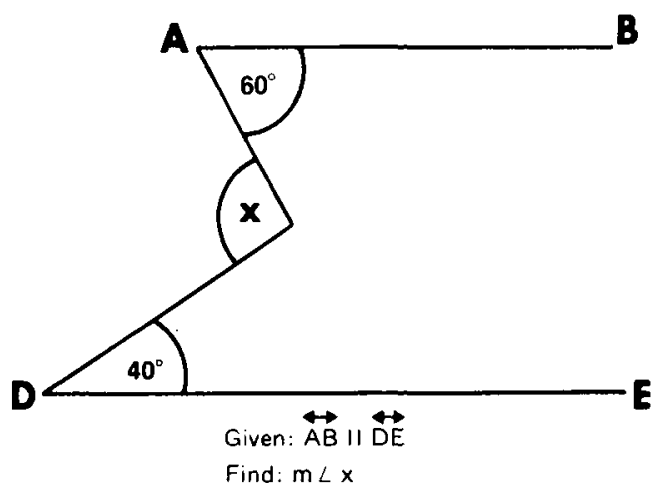

(b)

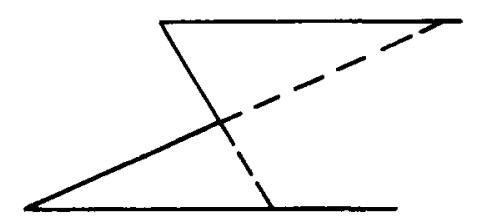

(c)

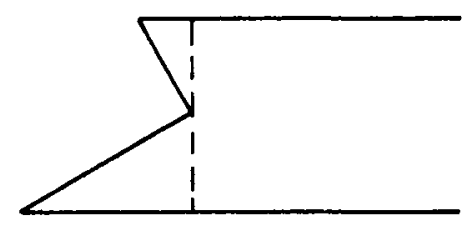

(d)

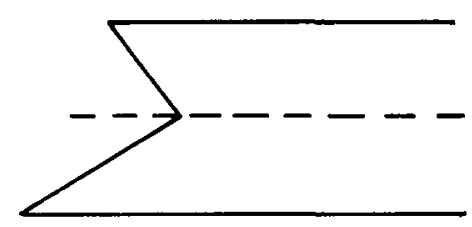

Figure 6. Problem involving parallel lines, and constructions formed by subjects.

induction of congruent triangles, Figure $5 \mathrm{c}$ was Problem 14; for the subjects with induction of parallel lines, Figure $5 \mathrm{~b}$ was Problem 14. This permitted comparison between subjects for whom the problem was consistent with the induction series and subjects for whom it was not.

\section{Results}

The manipulation was strong enough to produce reliable differences, even with only six subjects per condition. In the ambiguous problem, Figure 5a, five of the subjects who received induction with congruent triangles solved the problem by constructing the diagonal and proving that the resulting triangles were congruent. Only one of the six subjects who were induced to use parallel lines found the proof based on triangles; the other five found proofs using relations formed by parallel lines. The difference is significant $(p<.02)$ on a sign test for which the random event is agreement between the subject's solution and the induction sequence.

The problem in Figure 5c must be solved by proving that triangles are congruent. All six subjects who were induced for congruence of triangles adopted a plan 
involving triangles initially. In the group induced with parallel lines, only two subjects initially worked with a plan for proving the triangles congruent; the other four subjects tried to use the relation of parallel lines. This is also significant by a sign test $(p<.02)$

The problem in Figure $5 \mathrm{~b}$ is solved using parallel lines. In the group for which induction was with parallel lines, four of the six subjects used the plan involving parallel lines initially; the other two failed to see that parallel lines could be inferred but mentioned parallel lines as a subgoal. In the group for which induction was with congruent triangles, three of the six subjects began with a plan involving congruent triangles, and only one used parallel lines; the other two subjects initially used a plan involving the sum of the interior angles of a quadrilateral. Counting just those subjects whose initial plan was either congruence or parallel lines, the difference between the conditions was significant $(p<.04$ by sign test).

\section{Discussion}

This experiment on set provides a supportive illustration of the idea of a strong connection between the process of making constructions and the process of planning. The idea that a single planning process is responsible for both problem solving set and the making of constructions seems a plausible one.

\section{THINKING-ALOUD PROTOCOLS}

A collection of thinking-aloud protocols was obtained from six students who were interviewed individually approximately once each week during the year in which they took a course in high school geometry. In the interviews, students solved a wide variety of problems, only some of which are relevant to the issues in this paper. Solutions of nine of the problems used constructions, and two of the problems provided illustrations of problem solving set. Protocols from these problems were examined to provide an informal evaluation of the general hypothesis of schema-based planning processes. A brief summary of the conclusions of this informal analysis will be given here. A more detailed discussion of the protocols from the nine problems involving constructions is available in a technical report (Greeno, Note 6).

\section{Problems Involving Constructions}

Plan-based constructions. Nine problems that were given required construction of auxiliary lines. Most of the protocols indicated solution processes that were generally consistent with the kind of process programmed in Perdix, in which constructions are related to schemata that function as plans. Various schemata were involved in the different problems, including formation of triangles and other geometric figures, and the central angle of a circle.

An example involving the pattern of parallel lines intersected by a transversal is shown in Figure 6. This example has special interest because different constructions were used by subjects, based on different schemata. This problem was given to students early in the course, before they had studied congruence of triangles. They had been taught about relations between pairs of angles with parallel sides, such as corresponding angles, alternate interior angles, and so on. Constructions used in solving the problem are shown in Figures $6 b, 6 c$, and $6 \mathrm{~d}$. The construction shown in Figure $6 \mathrm{~b}$ completes transversals that intersect the parallel lines by extending lines already in the diagram. The protocol in which Figure $6 \mathrm{~b}$ occurred indicated that the subject probably completed the pattern and made justified inferences based on alternate interior angles and then realized that the presence of triangles permitted further inferences, which then led to the solution. The subject who gave the construction in Figure $6 c$ specified the added line as the perpendicular going through the vertex and apparently foresaw using the $90 \mathrm{deg}$ angles to permit inference of the remaining angles in the triangles based on the sum of angles in a triangle being $180 \mathrm{deg}$. At the time this problem was presented, the class had not studied triangles, so the sum of angles of a triangle was recalled from earlier study by the subjects who used Figures $6 \mathrm{~b}$ and $6 \mathrm{c}$. The construction in Figure $6 \mathrm{~d}$ permitted solution without using the triangle-sum property. This construction was not used by any subjects on the problem in Figure 6a, although one subject saw that it could be used when a pattern of three parallel lines was presented in another problem, and another subject used it in another construction problem involving parallel lines.

Working-forward pattern completion. While most of the protocols were consistent with Perdix's procedure for constructing auxiliary lines, several included significant features that could not be simulated by the kind of procedure that has been programmed. One process involves pattern completion that does not seem to be directly related to the problem goal. An example occurred in a problem of proving that if sides of a triangle are unequal, the angles opposite those sides also are unequal. This problem requires a construction to form an isosceles triangle inside the given triangle. One student made a construction similar to the one in Figure 1, bisecting the angle between the two specified sides of the triangle. It seems likely that features of the problem reminded the subject of the pattern involving two triangles containing the angles in the problem goal. However, that pattern is not a useful one for proving inequality of angles.

Procedures for making forward-working constructions have not been programmed for Perdix; however, they are quite straightforward. The kind of process needed could be represented either as single productions activated by features of the situation with actions that add the auxiliary lines or set goals for adding lines. An example relevant to the problem used in Experiments 1 and 2 would be a production in which the condition is an arc of a circle with known measure and the action is 
construction of the central angle corresponding to the arc.

In order for a construction produced in working forward to be useful, there must be a procedure for recognizing the results of the construction that may be helpful in achieving the goal of the problem. The planning process represented in the present version of Perdix is well suited for that purpose, since the planning procedure consists of a pattern recognition system for identifying features that are favorable for the available plans. Thus, if a forward-working production were to produce a new feature of the problem that could enable choice of a plan, the system would only have to invoke its planning system in the usual way for that new feature to be recognized and used. For example, in the problem used in Experiment 1, the construction of the central angle produces a triangle that has the unknown angle as one of its components. This configuration of features is then available for a planning process that has the form of a pattern recognition system.

A problem that must be solved in a system that includes forward chaining is that many inferences may be generated that are not useful in the problem. This would become especially critical in a system that permits use of auxiliary lines, since this greatly increases the number of potential objects about which inferences might be made. Nevins (Note 7) and Ullman (Note 8) have given some attention to this difficulty in systems for geometry theorem proving. Nevins' system restricts forward-chaining inferences to those that are related to generally useful subgoals that he called paradigms, such as finding congruent triangles. Ullman's system applies a general criterion of compactness of inferred information, such as the inference that a closed figure is a parallelogram, for setting aside the process of forward chaining. It seems quite likely that some mechanism such as these would keep a process of forward chaining under control in Perdix, especially if forward chaining were used only when none of the plans that the system has provide a basis for progress.

Stages in specifying constructions. Another feature of some protocols that would require extension of Perdix occurred when constructions had to be given some geometric property in addition to the spatial features used in drawing the line. A construction in geometry is often added in a single step, for example, by connecting two points in the diagram. However, some constructions are more complicated, requiring specification of some geometric property in addition to simply locating a line in the diagram. An example is the construction used in proving that base angles of isosceles triangles are congruent. In Table 1 there is a clear indication that the subject drew the line that completed the pattern of two triangles and then chose the specification of the line as the bisector of the angle at Point $\mathrm{C}$, rather than identifying the construction as the angle bisector initially.

Geometry constructions illustrate a general feature of problems in which material added to the problem space must satisfy constraints. In geometry, the constraints are specified in a set of theorems that assert that certain constructions are permitted. An example of such a permissive theorem is that an angle has exactly one bisector, which is used to justify construction of the bisector of an angle. Students are taught that an auxiliary line added to a diagram should not be either underdetermined or overdetermined. That is, just enough conditions should be specified for the line that exactly one line can be drawn to meet the conditions.

The process of forming a construction apparently has two major components. One is a process that motivates the construction; the other specifies properties of the construction that satisfy constraints. The analysis given in this paper has dealt mainly with the process of motivating a construction; protocols seem consistent with the idea that constructions are motivated by knowledge of patterns in plan-schemata that can be completed by adding auxiliary lines. The process of satisfying constraints is relatively simple in geometry, in which permissive theorems give a complete list of the conditions that may be used. When a problem solver encounters a situation in which an auxiliary line is desired, the construction theorems are consulted and the construction is added if the conditions of a permissive construction theorem are satisfied in the situation.

Three distinguishable patterns of forming constructions of this kind occurred in the protocols available for this study. In one pattern, found in two of the five protocols for the base angles theorem, subjects specified the construction completely at the outset. A second way of forming the construction shown in Table 1 and by one other subject involved drawing the line, then giving the geometric specification in relation to a goal of the problem. In this case, the goal is to generate components of triangles that can be proved congruent, and, by specifying the auxiliary line as an angle bisector, there are congruent angles. The third way of forming the construction involved drawing the line, then giving a geometric specification that was not related to the goal of the problem in any definite way. The subject who showed this pattern drew the line, then said, "I would probably say that this is a median." After completing the problem, the subject answered a question about the construction by saying, "All I wanted was just a name for a line that was going to give me two triangles."

In its present form, Perdix satisfies multiple constraints on constructions because its productions for construction have appropriate specifications as parts of their actions. This is inconsistent with most of the constructions observed in the protocols used in this study. Most of the time when an angle bisector or a line parallel or perpendicular to a given line was constructed, there was no evidence of special consideration given to the legitimacy of the construction. Of the three examples in which permissive theorems seemed to be used, one was consistent with the process used by Gelernter's (1953) system, in which the need for the construction arises as a subgoal, and there is explicit 
checking of the permissive theorems stored as a list to see whether a justification can be found. In the other two cases, a specification was apparently chosen in order to satisfy subgoals that were anticipated for a later stage of the problem. This kind of anticipatory planning has not been implemented in Perdix, but it is not incompatible with the general kind of planning mechanism that Perdix uses. In Sacerdoti's (1977) model, NOAH, unbound variables can be included in high-level components of plans, so that further analysis can be performed before deciding precisely how to specify the actions that will be performed.

\section{Problems Involving Set}

Two problems were given that could be solved either by ratios of sides of special right triangles or by congruence of triangles. Simpler solutions could be obtained using congruence, but the class topic at the time was properties of special triangles, and a majority of the solutions obtained used that approach.

These protocols give further illustrations of the idea that general approaches to problems can be influenced by recent use. The currency of the properties of special triangles in the geometry course apparently provided a context that was sufficient to induce a problem solving set for most of these subjects. On the other hand, examination of the protocols provided evidence against the specific form of planning that is used in Perdix to explain problem solving set. Perdix has plans associated with goals and checks these plans one at a time until one of them is selected. Performance by some subjects was more flexible, involving inference of some propositions in an apparent working-forward process and consideration of congruence of triangles. One subject, thinking retrospectively about solving a problem, said, "I wondered why you were giving me these things, and I went at the problem saying, "Well, let me try and use these things,' and I said, 'Well I give up.' Or, 'I give up, I see there's an easier way in my mind.' But I-I was working on it until I saw that things just came more naturally in another direction."

Performance of some human subjects would be simulated more accurately if Perdix's planning productions could be tested occasionally during problem solving. Plan-schemata could be represented as structures that have some probability of being activated at any time during problem solving, rather than only at the time a goal is activated. Effects of set could still be simulated if the probability of a plan-schema's activation depended on the strengths of relevant productions, and these strengths were influenced by factors such as recency of successful use of the various plans in the system (cf. Anderson, 1976; McDermott \& Forgy, 1977).

\section{GENERAL DISCUSSION}

This final section includes comments on the relation- ship of constructions to the general question of illstructured problem solving and creativity, and on the general issue of problem solving set.

\section{Constructions in IIl-Structured Problems}

A property of many ill-structured problems is that the problem space initially given in the problem is insufficient for the solution to be achieved. The initial situation may contain few if any of the components that are eventually generated and brought together in a solution of the problem. Examples that are often used include problems of composition, such as music, poetry, paintings, or scientific articles, in which the problem solver adopts the goal of composing something and creates most of the elements of the problem space, as well as their arrangement, in order to solve the problem.

Geometry problems are not poetry, of course, but problems with constructions seem to provide a nontrivial but manageable case in which the initial problem space must be enriched in order for the problem to be solved. In the model we have described in this paper, constructions are required when available problem solving plans have prerequisites that are not present in the situation. Then the prerequisites of a plan become a goal that the problem solver tries to achieve. Construction of the prerequisites is itself a well-structured problem. However, solution of this problem requires operators (drawing lines) that are not in the set of operators available in the problem space of the main problem. ${ }^{2}$

In this analysis, constructions occur as solutions to auxiliary problems that arise in solving the main problem. This view puts problems requiring constructions into the class of problems requiring more than one problem space in their solution. Simon (1973) discussed general characteristics of problem solving with multiple problem spaces and pointed out that solutions require access to information that is used to generate new subgoals, new constraints, and new problem solving operators.

For ill-structured problems of this kind, the theory of well-structured problem solving must be extended by providing a mechanism for coordinating activity in different problem spaces. In Perdix, coordination is achieved by use of general schemata that guide pattern recognition and pattern completion processes of different kinds. A new problem space is generated when a pattern required by the planning process in one problem space is incomplete. Then, problem solving activity is transferred to a problem space that includes operators appropriate for the kind of pattern completion that is required.

The general nature of this mechanism seems a plausible basis for productive problem solving in many situations that involve composition. It seems a reasonable conjecture that an important component of composition is a process of pattern completion guided by the composer's knowledge of general schemata that represent general forms of material that is included in 
the work at various levels of structure. Flower and Hayes' (in press) analysis of planning processes in writing seems consistent with this general idea.

This idea also provides an intriguing possibility regarding an important aspect of creativity. Investigators who have studied creative problem solving in artistic composition have emphasized the importance of processes that result in the formulation of interesting problems. Apparently, skill in problem formulation is important in distinguishing artists whose work is recognized in the field from those who are competent, but not influential (Getzels \& Csikszentmihalyi, 1976). The analysis presented here suggests that the ability to formulate problems may be based on knowledge in the form of general schemata and procedures for recognizing the possibility of creating specific instances and combinations of general structures.

The general conclusion of this analysis is that in an ill-structured problem with an insufficient initial problem space, problem solving takes place in a number of different problem spaces. The contents of the initial problem space depend on the problem solver's knowledge about the domain, so problems that are ill structured for some problem solvers may be well structured for more experienced problem solvers. An important feature of ill-structured problems is that new problem spaces must be generated in the process of problem solving. There are probably many ways in which this occurs, but the mechanism that is in the present version of Perdix, involving generation of problems based on schemata that are also the basis of planning, seems to provide an interesting example of the process.

It should be remembered that the issue of ill-structured problems involves other factors in addition to insufficient initial problem spaces. Another way in which a problem can have weak structure is to have an indefinite goal that can be achieved by alternative combinations of components or features. The idea of an indefinite goal is represented in Perdix as a pattern recognition system that identifies the various combinations of congruent components that are sufficient to prove that triangles are congruent (Greeno, 1976). Since it can work both with indefinite goals and incomplete initial problem spaces, Perdix seems to provide a moderately successful extension of problem solving theory into the domain of ill-structured problems. Of course, there are important aspects of ill-structured problem solving that are not represented in Perdix, such as the development of new problem solving operators and the discovery of new concepts of strategies in the course of problem solving. Recent analyses of learning processes in problem solving systems (Anzai \& Simon, 1979; Neches \& Hayes, 1978; Sussman, 1975) suggest that these generative aspects of creative problem solving may be understood best as instances of learning.

\section{Genceral Nature of Set}

The traditional behaviorist explanation of set is that a higher level response that has high strength dominates performance on the problem. The explanation in Perdix is consistent with this idea and adds a specific mechanism by which higher level responses can have their dominating effect. The explanation gains in interest because the cognitive structure that causes set is the same as the structure that permits constructions. It is of some interest to consider the relationship between the mechanism proposed here and various phenomena of problem solving set that have been investigated experimentally.

There are two general kinds of problem solving set. One kind is typified by Luchins' (1942) water-jug tasks and the examples from geometry discussed in this paper, in which the problem solver is biased toward the use of a problem solving plan or method. A second kind of phenomenon is involved in functional fixedness, studied by Duncker (1945), in which use of an object or idea in one context prevents the problem solver from recognizing a potential use of that object or idea in a subsequent problem situation.

Biased top-down planning. The principle of schemabased planning represented in Perdix gives a straightforward explanation of problem solving set that involves bias toward use of a specific problem solving method. For example, a system such as Perdix involving schemata for solution types in Luchins' (1942) water-jug task would show biases of the same kind as it shows for the geometry problems considered in this paper, in agreement with performance of human subjects.

Lewis (1978) has also developed an analysis of set to use a specific problem solving method. In Lewis' system, a sequence of actions that occurs regularly can become integrated into a single action. This integration can eliminate tests for appropriateness of intermediate steps in the solution. Without performing these tests, the problem solver may miss opportunities to solve the problem in a more efficient way, as in the situation studied by Luchins (1942). Using a letter-substitution task, Lewis obtained evidence that subjects integrated problem solving steps in a way consistent with his model, but they maintained a capability of noticing conditions for applying alternative operations when they were instructed to use the alternatives if possible. It seems possible that Lewis' system could provide an explanation for the development of structures that correspond to plans in the version of Perdix described in this paper. Plans are integrated action sequences, but, in general, use of a plan is optional, so that with appropriate instructions, a plan's inappropriate use could be avoided.

Functional fixedness. Phenomena involving functional fixedness seem to be of a different character from those involving a specific problem solving method. Weisberg and Suls' (1973) analysis of the candle problem and Magone's (1977) analysis of the two-string problem identified analysis of features of objects in the situation as an important component of the process of solving the problem. For example, in the candle problem, in 
which a box must be seen as a potential supporting surface, a condition for solution may be the problem solver's noticing the flatness of the box. Magone found that subjects achieved a greater variety of solutions in the two-string problem if they were initially prompted to consider features of objects than if they were initially prompted to find a solution of a specified kind, such as extension of one of the strings or causing a string to swing back and forth.

There are two alternative mechanisms that could be postulated in the framework of a production system for explaining functional fixedness, viewed as prevention of recognizing some feature of an object in the situation. One mechanism would cause functional fixedness by engaging in a top-down search for objects with features that could be used in a plan-schema that was previously selected. For example, if the system had selected the plan of extending a string in the two-string problem, then the analysis of objects in the situation would involve their lengths rather than other features that would fit into different possible plans for solving the problem. A second mechanism would involve competing productions for identifying features of objects. Suppose that an object has two features that can be identified by different productions, and one of these is much stronger than the other because of the way the object has already been used in the situation. Then, when that object is considered by the problem solver, the production that could identify the second feature may be prevented from executing by the rapid execution of the stronger production.

The mechanism of top-down search is very similar to the mechanism that is represented in Perdix to provide an explanation for problem solving set based on top-down planning. Consider Figure 2 and recall that Perdix solves this problem by proving that triangles are congruent. The solution involves recognizing the presence of triangles in the diagram, rather than another feature that is also present, the fact that the two angles involved in the goal of the problem have the same vertex. Perdix fails to notice a relevant feature of the problem because a general plan-schema that includes tests for different features is active and prevents testing for the feature of a shared vertex. A simulation of problem solving in situations such as the candle or two-string problems would require a somewhat different use of this kind of structure, since there are several different objects in the situation, and, in some cases, failure to find a feature of an object needed for the current plan apparently leads the problem solver to pass by that object rather than to test the object for additional features. However, it seems reasonable to conjecture that the basic structure of schema-based planning that causes Perdix to show problem solving set in geometry would also produce functional fixedness in situations in which attempts to use a general plan lead to the neglect of important features of objects.

Phenomena recently reported by Halstead-Nussloch (1978) can be interpreted by assuming a top-down search for problem features. Halstead-Nussloch trained different groups of subjects to identify serial patterns using two different pattern languages taken from analyses by Restle (1970) and Simon (1972). Results indicated that subjects trained to represent serial patterns in terms of hierarchies of transformations applied that schema in the analysis of sequences that could be analyzed either as hierarchies of transformations or as sequences composed of two or more interleaved subsequences. Another kind of set phenomenon that can be interpreted as resulting from top-down search is the difficulty of simple discrimination learning following a few trials of random feedback, reported by Levine (1971). Levine's interpretation was that, during random feedback trials, subjects rejected simple hypotheses about stimulus attributes and removed them from consideration and thus were prevented from recognizing simple relationships between stimulus attributes and feedback on later trials. Search for solution was apparently guided by a metahypothesis that the correct rule was a sequential pattern, which permits a very large number of possibilities. Plans, as represented in Perdix, establish categories of hypotheses that guide search for a solution to a problem, and thus planning processes provide a specific procedural hypothesis with which to understand Levine's findings.

The second mechanism, in which use of an object in one way causes a feature of the object to be ignored, probably involves a different functional level of productions from the planning procedures that we have considered in this paper. Some cases in which a feature of an object is ignored probably involve simple perceptual salience. In the candle problem, in which use of a box as a candlestand is inhibited by its use for holding tacks, solution is facilitated if the word "box" is printed on the box, relative to the situation in which "tacks" is printed on the box (Glucksberg \& Weisberg, 1966). This presumably aids recognition of the physical features of the box through simple direction of attention. A similar phenomenon has recently been reported by Sweller and Gee (1978), who found that effects of set in Luchins-type water-jug problems (Luchins, 1942) and Levine-type induction problems (Levine, 1971) were greatly reduced by perceptual manipulations. In the water-jug situation, Sweller and Gee virtually eliminated the effect of set by having subjects write their solutions numerically, rather than in terms of letters designating the jars used. In induction problems, the manipulation involved giving feedback that described the correct stimulus, rather than stating whether the subject's response was correct or incorrect. Both of these manipulations seem to be interpretable as avoiding the removal of attention from aspects of the situation that are important in solving a problem, and they thus support the hypothesis that set occurs because of relatively low salience of the important stimulus features.

In other cases, however, suppression of detection of a feature is apparently related to a more active process. 
In Duncker's (1945) gimlet problem, use of the gimlet as a hook was inhibited by the subject's use of the gimlet to bore holes for the conventional hooks that were available. In a production system, solution of the problem of boring holes would strengthen productions for identifying features of the gimlet used in boring holes and could make less likely the recognition of features involved in serving as a hook.

All of the explanations of functional fixedness proposed here are based on the idea that productions must be executed for the relevant features of objects to be recognized. The required productions can be prevented by the dominance of other recognition productions, involving top-down search, ordinary perceptual salience, or recent use in another context.

\section{REFERENCE NOTES}

1. Goldstein, I. P. Elementary geometry theorem proving (Artificial Intelligence Memo 280). Cambridge, Mass: M.I.T., 1973.

2. Larkin, J. H. Skilled problem solving in physics: $A$ hierarchical planning model. Unpublished manuscript, University of California at Berkeley, September 1977.

3. Atwood, M. E., Polson, P. G., Jeffries, R., \& Ramsey, H. R. Planning as a process of synthesis (Tech. Rep. SAI-78-144-DEN). Englewood, Colo: Science Applications Inc., December 1978.

4. Goldstein, I. P., \& Miller, M. L. Structured planning and debugging a linguistic theory of design (Artificial Intelligence Memo 387). Cambridge, Mass: M.I.T., Artificial Intelligence Laboratory, December 1976.

5. Long, W. J. A program writer (Tech. Rep. MIT/LCS/TR-

187). Cambridge, Mass: M.I.T., Laboratory for Computer Science, November 1977.

6. Greeno, J. G. Constructions in geometry problem solving (Tech. Rep.). Pittsburgh, Pa: University of Pittsburgh, Learning Research and Development Center, in press.

7. Nevins, A. J. Plane geometry theorem proving using forward chaining (Artificial Intelligence Memo 303). Cambridge, Mass: M.I.T., January 1974.

8. Ullman, S. A model-driven geometry theorem prover (Artificial Intelligence Memo 321). Cambridge, Mass: M.I.T., May 1975.

\section{REFERENCES}

ANDERSON, J. R. Language, memory, and thought. Hillsdale, N.J: Erlbaum, 1976.

Anzal, Y., \& Simon, H. A. The theory of learning by doing. Psychological Review, 1979, 86, 124-140.

BerLyNe, D. E. Structure and direction in thinking. New York: Wiley, 1965.

Bhaskar, R., \& Simon, H. A. Problem solving in semantically rich domains: An example from engineering thermodynamics. Cognitive Science, 1977, 1, 193-215.

Bourne, L. E., Jr., Ekstrand, B. R., \& Dominowski, B. R. The psychology of thinking. Englewood Cliffs, N.J: PrenticeHall, 1971.

Duncker, K. On problem solving. Psychological Monographs, 1945,58 (Whole No. 270).

Flower, L., \& Hayes, J. R. Plans that guide the composing process. In C. Frederiksen, M. Whiteman, \& J. Dominic (Eds.), Writing: The nature, development and teaching of written communication. Hillsdale, N.J: Erlbaum, in press.
Gelernte R, H. Realization of a geometry-theorem proving machine. In E. A. Feigenbaum \& J. Feldman (Eds.), Computers and thought. New York: McGraw-Hill, 1963.

Getzels, J. W., \& Csikszentminalyi, M. The creative vision: A longitudinal study of problem finding in art. New York: Wiley, 1976.

Glucksberg, S., \& Weisberg, R. W. Verbal behavior and problem solving: Some effects of labeling in a functional fixedness problem. Journal of Experimental Psychology, 1966, 71, 659-664.

GreEno, J. G. Indefinite goals in well-structured problems. Psychological Review, 1976, 83, 479-491.

Greeno, J. G. Process of understanding in problem solving. In N. J. Castellan, D. B. Pisoni, \& G. R. Potts (Eds.), Cognitive theory (Vol. 2). Hillsdale, N.J: Erlbaum, 1977.

Greeno, J. G. A study of problem solving. In R. Glaser (Ed.), Advances in instructional psychology (Vol. 1). Hillsdale, N.J: Erlbaum, 1978.

Halste Ad-Nussloch, R. P. Characteristics of set in sequence learning. Unpublished doctoral dissertation, University of Michigan, 1978.

HuLl, C. L. Knowledge and purpose as habit mechanisms. Psychological Review, 1930, 37, 241-256.

Levine, M. Hypothesis theory and nonlearning despite ideal S-R-reinforcement contingencies. Psychological Review, 1971, 78, 130-140.

LEWIS, C. H. Production system models of practice effects. Unpublished doctoral dissertation, University of Michigan, 1978.

Luchins, A. S. Mechanization in problem solving. Psychological Monographs, 1942, 54(Whole No. 248).

MAGONE, M. E. Goal analysis and feature detection as processes in the solution of an insight problem. Unpublished master's thesis, University of Pittsburgh, 1977.

McDermott, D. Planning and acting. Cognitive Science, 1978, 2, 71-110.

McDermott, J., \& Forgy, C. Production system conflict resolution strategies. In D. Waterman \& F. Hayes-Roth (Eds.), Pattern-directed inference systems. New York: Academic Press, 1977.

Miller, G. A., Galanter, E. H., \& Pribram, K. H. Plans and the structure of behavior. New York: Holt, Rinehart, \& Winston, 1960.

Neches, R., \& Hayes, J. R. Progress towards a taxonomy of strategy transformations. In A. M. Lesgold, J. W. Pellegrino, S. D. Fokkema, \& R. Glaser (Eds.), Cognitive psychology and instruction. New York: Plenum Press, 1978.

Reitman, W. R. Cognition and thought. New York: Wiley, 1965.

Restle, F. Theory of serial pattern learning: Structural trees. Psychological Review, 1970, 77, 481-495.

Sace RDoti, E. D. A structure for plans and behavior. New York: Elsevier-North Holland, 1977.

Simon, H. A. Complexity and the representation of patterned sequences of symbols. Psychological Review, 1972, 79, 369-382.

Simon, H. A. The structure of ill structured problems. Artificial Intelligence, 1973, 4, 181-201.

Sussman, G. J. A computer model of skill acquisition. New York: Elsevier-North Holland, 1975.

Sussman, G. J. Electrical design: A problem for artificial intelligence research. Proceedings of the International Joint Conference on Artificial Intelligence. Cambridge, Mass: 1977.

Sweller, J., \& GeE, W. Einstellung, the sequence effect, and hypothesis theory. Journal of Experimental Psychology: Human Learning and Memory, 1978, 4, 513-526.

Weisberg, R., \& Suls, J. M. An information-processing model of Duncker's candle problem. Cognitive Psychology, 1973, 4, 255-276. 


\section{NOTES}

1. The part of the system in which the productions for constructions interact with the present planning system has not been debugged. At its present size, the system is relatively expensive to work with, and I believe that only technical insights would be gained from completing the programming task. The productions for construction were debugged in an earlier system, described at meetings of the Midwestem Psychological Association in 1976, in which the decision to construct a line was made when conditions for setting a subgoal of congruent triangles were satisfied.

2. This emphasizes that a problem is well or ill structured only in relation to a specified problem space. In the specification assumed here, problem solving operators are the propositions of geometry that permit inferences about properties and relations, and operators that permit drawing lines are in another problem space. Of course, an expanded problem space could be specified in which both sets of operators were available. In that case, problems requiring constructions would be well defined in the usual sense, and the construction of auxiliary lines would be guided by ordinary subgoals.

(Received for publication November 15, 1978; revision accepted August 25, 1979.) 